\title{
Coefficient Bounds for Certain Subclasses of Bi-Univalent Function
}

\author{
G. Murugusundaramoorthy, ${ }^{1}$ N. Magesh, $^{2}$ and V. Prameela ${ }^{3}$ \\ ${ }^{1}$ School of Advanced Sciences, VIT University, Vellore, Tamil Nadu 632014, India \\ ${ }^{2}$ PG and Research Department of Mathematics, Government Arts College (Men), Krishnagiri, Tamil Nadu 635001, India \\ ${ }^{3}$ Department of Mathematics, Adhiyamaan College of Engineering (Autonomous), Hosur, Tamil Nadu 635109, India
}

Correspondence should be addressed to G. Murugusundaramoorthy; gmsmoorthy@yahoo.com

Received 11 February 2013; Accepted 23 May 2013

Academic Editor: Pavel Kurasov

Copyright (c) 2013 G. Murugusundaramoorthy et al. This is an open access article distributed under the Creative Commons Attribution License, which permits unrestricted use, distribution, and reproduction in any medium, provided the original work is properly cited.

We introduce two new subclasses of the function class $\Sigma$ of bi-univalent functions defined in the open unit disc. Furthermore, we find estimates on the coefficients $\left|a_{2}\right|$ and $\left|a_{3}\right|$ for functions in these new subclasses. Also consequences of the results are pointed out.

\section{Introduction}

Denote by $\mathscr{A}$ the class of functions of the form

$$
f(z)=z+\sum_{n=2}^{\infty} a_{n} z^{n}
$$

which are analytic in the open unit disc $\mathbb{U}=\{z: z \in$ $\mathbb{C}$ and $|z|<1\}$. Further, denote by $\mathcal{S}$ the class of all functions in $\mathscr{A}$ which are univalent and normalized by $f(0)=0=$ $f^{\prime}(0)$ in $\mathbb{U}$. The well-investigated subclasses of the univalent function class $\mathcal{S}$ are the class of starlike functions of order $\alpha(0 \leq \alpha<1)$, denoted by $\mathcal{S}^{*}(\alpha)$ and the class of convex functions of order $\alpha$ denoted by $\mathscr{K}(\alpha)$ in $\mathbb{U}$. It is well known that every function $f \in \mathcal{S}$ has an inverse $f^{-1}$, defined by

$$
\begin{gathered}
f^{-1}(f(z))=z, \quad z \in \mathbb{U}, \\
f\left(f^{-1}(w)\right)=w, \quad|w|<r_{0}(f), \quad r_{0}(f) \geq \frac{1}{4},
\end{gathered}
$$

where

$$
\begin{aligned}
f^{-1}(w)= & w-a_{2} w^{2}+\left(2 a_{2}^{2}-a_{3}\right) w^{3} \\
& -\left(5 a_{2}^{3}-5 a_{2} a_{3}+a_{4}\right) w^{4}+\cdots .
\end{aligned}
$$

A function $f \in \mathscr{A}$ is said to be bi-univalent in $\mathbb{U}$ if both $f(z)$ and $f^{-1}(z)$ are univalent in $\mathbb{U}$. Let $\Sigma$ denote the class of bi-univalent functions in $\mathbb{U}$ given by (1).
Analogous to the function class $\mathcal{S}$, the bi-univalent function class $\Sigma$ include, for example, the class $\delta_{\Sigma}^{*}(\alpha)$ of bistarlike functions of order $\alpha(0 \leq \alpha<1)$, the class $\mathscr{K}_{\Sigma}(\alpha)$ of biconvex functions of order $\alpha(0 \leq \alpha<1)$, and the class $\delta_{\Sigma}^{\alpha}$ of strongly bi-starlike functions of order $\alpha(0<$ $\alpha \leq 1)$. For some intriguing examples of functions and characterization of the class $\Sigma$, one could refer to Srivastava et al. [1] and the references stated therein (see also [2]). Recently there has been triggering, interest to study the biunivalent functions class $\Sigma$ (see [2-5]) and obtain nonsharp estimates on the first two Taylor-Maclaurin coefficients $\left|a_{2}\right|$ and $\left|a_{3}\right|$. The coefficient estimate problem for each of the following Taylor-Maclaurin coefficients $\left|a_{n}\right|$ for $n \in \mathbb{N} \backslash\{1,2\}$, $\mathbb{N}:=\{1,2,3, \ldots\}$ is presumably still an open problem.

Motivated by the earlier works of Srivastava et al. [1] and Frasin and Aouf [3] in the present paper we introduce the following two new subclasses of the function class $\Sigma$.

Definition 1. A function $f(z)$ given by (1) is said to be in the class $\mathscr{G}_{\Sigma}(\alpha, \lambda)$ if the following conditions are satisfied:

$$
\begin{array}{r}
f \in \Sigma, \quad\left|\arg \left(\frac{z f^{\prime}(z)}{(1-\lambda) f(z)+\lambda z f^{\prime}(z)}\right)\right|<\frac{\alpha \pi}{2}, \\
0<\alpha \leq 1,0 \leq \lambda<1, \quad z \in \mathbb{U},
\end{array}
$$




$$
\begin{array}{r}
\left|\arg \left(\frac{w g^{\prime}(w)}{(1-\lambda) g(w)+\lambda w g^{\prime}(w)}\right)\right|<\frac{\alpha \pi}{2}, \\
0<\alpha \leq 1,0 \leq \lambda<1, w \in \mathbb{U},
\end{array}
$$

where the function $g$ is given by

$$
\begin{aligned}
g(w)= & w-a_{2} w^{2}+\left(2 a_{2}^{2}-a_{3}\right) w^{3} \\
& -\left(5 a_{2}^{3}-5 a_{2} a_{3}+a_{4}\right) w^{4}+\cdots
\end{aligned}
$$

Definition 2. A function $f(z)$ given by (1) is said to be in the class $\mathscr{M}_{\Sigma}(\beta, \lambda)$ if the following conditions are satisfied:

$$
\begin{gathered}
f \in \Sigma, \quad \mathfrak{R}\left(\frac{z f^{\prime}(z)}{(1-\lambda) f(z)+\lambda z f^{\prime}(z)}\right)>\beta, \\
0 \leq \beta<1,0 \leq \lambda<1, z \in \mathbb{U}, \\
\Re\left(\frac{w g^{\prime}(w)}{(1-\lambda) g(w)+\lambda w g^{\prime}(w)}\right)>\beta, \\
0 \leq \beta<1,0 \leq \lambda<1, w \in \mathbb{U},
\end{gathered}
$$

where the function $g$ is given by (5).

It is of interest to note that, for $\lambda=0$, the class $\mathscr{G}_{\Sigma}(\alpha, \lambda)$ reduces to $\mathcal{S}_{\Sigma}^{\alpha}$ of strongly bi-starlike functions of order $\alpha$ and the class $\mathscr{M}_{\Sigma}(\beta, \lambda)$ leads to $\mathcal{S}_{\Sigma}^{*}(\beta)$ bi-starlike functions of order $\beta$.

The object of the present paper is to find estimates on the coefficients $\left|a_{2}\right|$ and $\left|a_{3}\right|$ for functions in the above-defined subclasses $\mathscr{G}_{\Sigma}(\alpha, \lambda)$ and $\mathscr{M}_{\Sigma}(\beta, \lambda)$ of the function class $\Sigma$ by employing the techniques used earlier by Srivastava et al. [1]. lemma.

In order to derive our main results, we recall the following

Lemma 3 (see [6]). If $\in \mathscr{P}$, then $\left|c_{k}\right| \leq 2$ for each $k$, where $\mathscr{P}$, is the family of all functions $h$ analytic in $\mathbb{U}$ for which $\mathfrak{R}\{h(z)\}>$ 0 , where $h(z)=1+c_{1} z+c_{2} z^{2}+\cdots$ for $z \in \mathbb{U}$.

\section{Coefficient Bounds for the Function$$
\text { Class } \mathscr{G}_{\Sigma}(\alpha, \lambda)
$$

We begin by finding the estimates on the coefficients $\left|a_{2}\right|$ and $\left|a_{3}\right|$ for functions in the class $f \in \mathscr{G}_{\Sigma}(\alpha, \lambda)$.

Theorem 4. Let $f(z)$ given by (1) be in the class $\mathscr{G}_{\Sigma}(\alpha, \lambda), 0<$ $\alpha \leq 1$, and $0 \leq \lambda<1$. Then

$$
\begin{gathered}
\left|a_{2}\right| \leq \frac{2 \alpha}{(1-\lambda) \sqrt{1+\alpha}}, \\
\left|a_{3}\right| \leq \frac{4 \alpha^{2}}{(1-\lambda)^{2}}+\frac{\alpha}{1-\lambda} .
\end{gathered}
$$

Proof. It follows from (4) that

$$
\begin{aligned}
& \frac{z f^{\prime}(z)}{(1-\lambda) f(z)+\lambda z f^{\prime}(z)}=[p(z)]^{\alpha}, \\
& \frac{w g^{\prime}(w)}{(1-\lambda) g(w)+\lambda w g^{\prime}(w)}=[q(w)]^{\alpha},
\end{aligned}
$$

where $p(z)$ and $q(w)$ in $\mathscr{P}$ have the forms

$$
\begin{aligned}
& p(z)=1+p_{1} z+p_{2} z^{2}+\cdots, \\
& q(z)=1+q_{1} w+q_{2} w^{2}+\cdots
\end{aligned}
$$

Now, equating the coefficients in (9), we get

$$
\begin{gathered}
(1-\lambda) a_{2}=\alpha p_{1}, \\
\left(\lambda^{2}-1\right) a_{2}^{2}+2(1-\lambda) a_{3} \\
=\frac{1}{2}\left[\alpha(\alpha-1) p_{1}^{2}+2 \alpha p_{2}\right], \\
-(1-\lambda) a_{2}=\alpha q_{1}, \\
\left(\lambda^{2}-4 \lambda+3\right) a_{2}^{2}-2(1-\lambda) a_{3} \\
=\frac{1}{2}\left[\alpha(\alpha-1) q_{1}^{2}+2 \alpha q_{2}\right] .
\end{gathered}
$$

From (12) and (14), we get

$$
\begin{aligned}
p_{1} & =-q_{1}, \\
2(1-\lambda)^{2} a_{2}^{2} & =\alpha^{2}\left(p_{1}^{2}+q_{1}^{2}\right) .
\end{aligned}
$$

From (13), (15), and (17), we obtain

$$
a_{2}^{2}=\frac{\alpha^{2}\left(p_{2}+q_{2}\right)}{(\alpha+1)(1-\lambda)^{2}} .
$$

Applying Lemma 3 for the coefficients $p_{2}$ and $q_{2}$, we immediately have

$$
\left|a_{2}\right| \leq \frac{2 \alpha}{(1-\lambda) \sqrt{(1+\alpha)}}
$$

This gives the bound on $\left|a_{2}\right|$ as asserted in (7).

Next, in order to find the bound on $\left|a_{3}\right|$, by subtracting (15) from (13), we get

$$
\begin{aligned}
& 4(1-\lambda) a_{3}-4(1-\lambda) a_{2}^{2} \\
& \quad=\alpha\left(p_{2}-q_{2}\right)+\frac{\alpha(\alpha-1)}{2}\left(p_{1}^{2}-q_{1}^{2}\right) .
\end{aligned}
$$

It follows from (16), (17), and (20) that

$$
a_{3}=\frac{\alpha\left(p_{2}-q_{2}\right)}{4(1-\lambda)}+\frac{\alpha^{2}\left(p_{1}^{2}+q_{1}^{2}\right)}{2(1-\lambda)^{2}} .
$$


Applying Lemma 3 once again for the coefficients $p_{1}, p_{2}$, $q_{1}$, and $q_{2}$, we readily get

$$
\left|a_{3}\right| \leq \frac{4 \alpha^{2}}{(1-\lambda)^{2}}+\frac{\alpha}{1-\lambda} .
$$

This completes the proof of Theorem 4 .

In the following section we find the estimates on the coefficients $\left|a_{2}\right|$ and $\left|a_{3}\right|$ for functions in the class $\mathscr{M}_{\Sigma}(\beta, \lambda)$.

\section{Coefficient Bounds for the Function}

Class $\mathscr{M}_{\Sigma}(\beta, \lambda)$

Theorem 5. Let $f(z)$ given by $(1)$ be in the class $\mathscr{M}_{\Sigma}(\beta, \lambda), 0 \leq$ $\beta<1$, and $0 \leq \lambda<1$. Then

$$
\begin{gathered}
\left|a_{2}\right| \leq \frac{\sqrt{2(1-\beta)}}{1-\lambda}, \\
\left|a_{3}\right| \leq \frac{4(1-\beta)^{2}}{(1-\lambda)^{2}}+\frac{(1-\beta)}{1-\lambda} .
\end{gathered}
$$

Proof. It follows from (6) that there exists $p, q \in \mathscr{P}$ such that

$$
\begin{aligned}
& \frac{z f^{\prime}(z)}{(1-\lambda) f(z)+\lambda z f^{\prime}(z)}=\beta+(1-\beta) p(z), \\
& \frac{w g^{\prime}(w)}{(1-\lambda) g(w)+\lambda w g^{\prime}(w)}=\beta+(1-\beta) q(w),
\end{aligned}
$$

where $p(z)$ and $q(w)$ have the forms of (10) and (11), respectively. Equating coefficients in (24) we get

$$
\begin{gathered}
(1-\lambda) a_{2}=(1-\beta) p_{1}, \\
\left(\lambda^{2}-1\right) a_{2}^{2}+2(1-\lambda) a_{3}=(1-\beta) p_{2}, \\
-(1-\lambda) a_{2}=(1-\beta) q_{1}, \\
\left(\lambda^{2}-4 \lambda+3\right) a_{2}^{2}-2(1-\lambda) a_{3}=(1-\beta) q_{2} .
\end{gathered}
$$

The proof follows, by employing the techniques used in the proof of Theorem 4 .

Taking $\lambda=0$ in Theorems 4 and 5 one can get the following corollaries.

Corollary 6. Let $f(z)$ given by (1) be in the class $\delta_{\Sigma}^{\alpha}$ and $0<$ $\alpha \leq 1$. Then

$$
\left|a_{2}\right| \leq \frac{2 \alpha}{\sqrt{\alpha+1}}, \quad\left|a_{3}\right| \leq 4 \alpha^{2}+\alpha
$$

Corollary 7. Let $f(z)$ given by $(1)$ be in the class $\mathcal{S}_{\Sigma}^{*}(\beta)$ and $0 \leq \beta<1$. Then

$$
\left|a_{2}\right| \leq \sqrt{2-2 \beta}, \quad\left|a_{3}\right| \leq 4(1-\beta)^{2}+(1-\beta) .
$$

\section{Acknowledgment}

The authors would like to record their sincere thanks to the referees for their valuable suggestions.

\section{References}

[1] H. M. Srivastava, A. K. Mishra, and P. Gochhayat, "Certain subclasses of analytic and bi-univalent functions," Applied Mathematics Letters, vol. 23, no. 10, pp. 1188-1192, 2010.

[2] T. Hayami and S. Owa, "Coefficient bounds for bi-univalent functions," Pan-American Mathematical Journal, vol. 22, no. 4, pp. 15-26, 2012.

[3] B. A. Frasin and M. K. Aouf, "New subclasses of bi-univalent functions," Applied Mathematics Letters, vol. 24, no. 9, pp. 15691573, 2011.

[4] Q.-H. Xu, Y.-C. Gui, and H. M. Srivastava, "Coefficient estimates for a certain subclass of analytic and bi-univalent functions," Applied Mathematics Letters, vol. 25, no. 6, pp. 990-994, 2012.

[5] Q.-H. Xu, H.-G. Xiao, and H. M. Srivastava, "A certain general subclass of analytic and bi-univalent functions and associated coefficient estimate problems," Applied Mathematics and Computation, vol. 218, no. 23, pp. 11461-11465, 2012.

[6] C. Pommerenke, Univalent Functions, Vandenhoeck \& Ruprecht, Göttingen, Germany, 1975. 


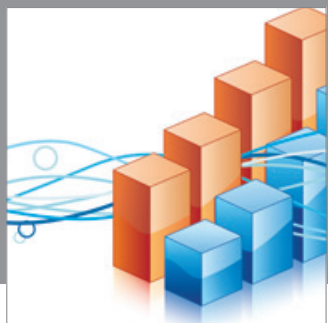

Advances in

Operations Research

mansans

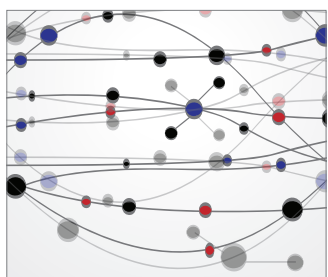

The Scientific World Journal
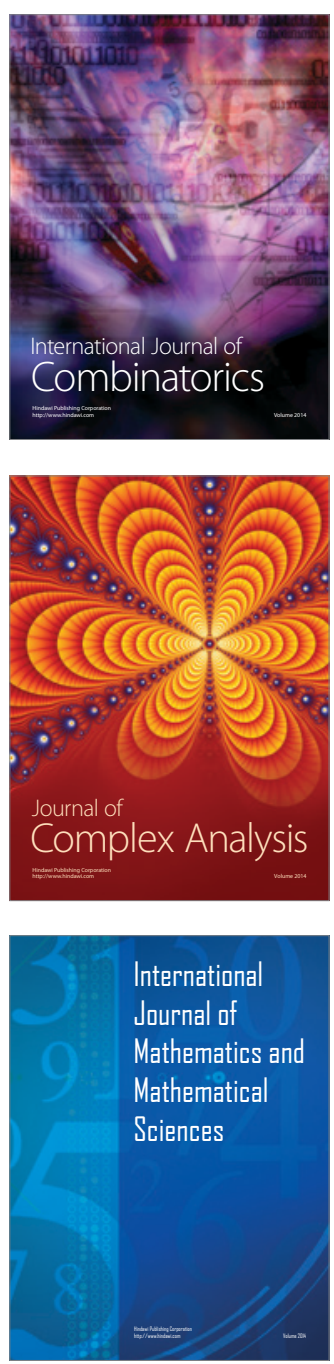
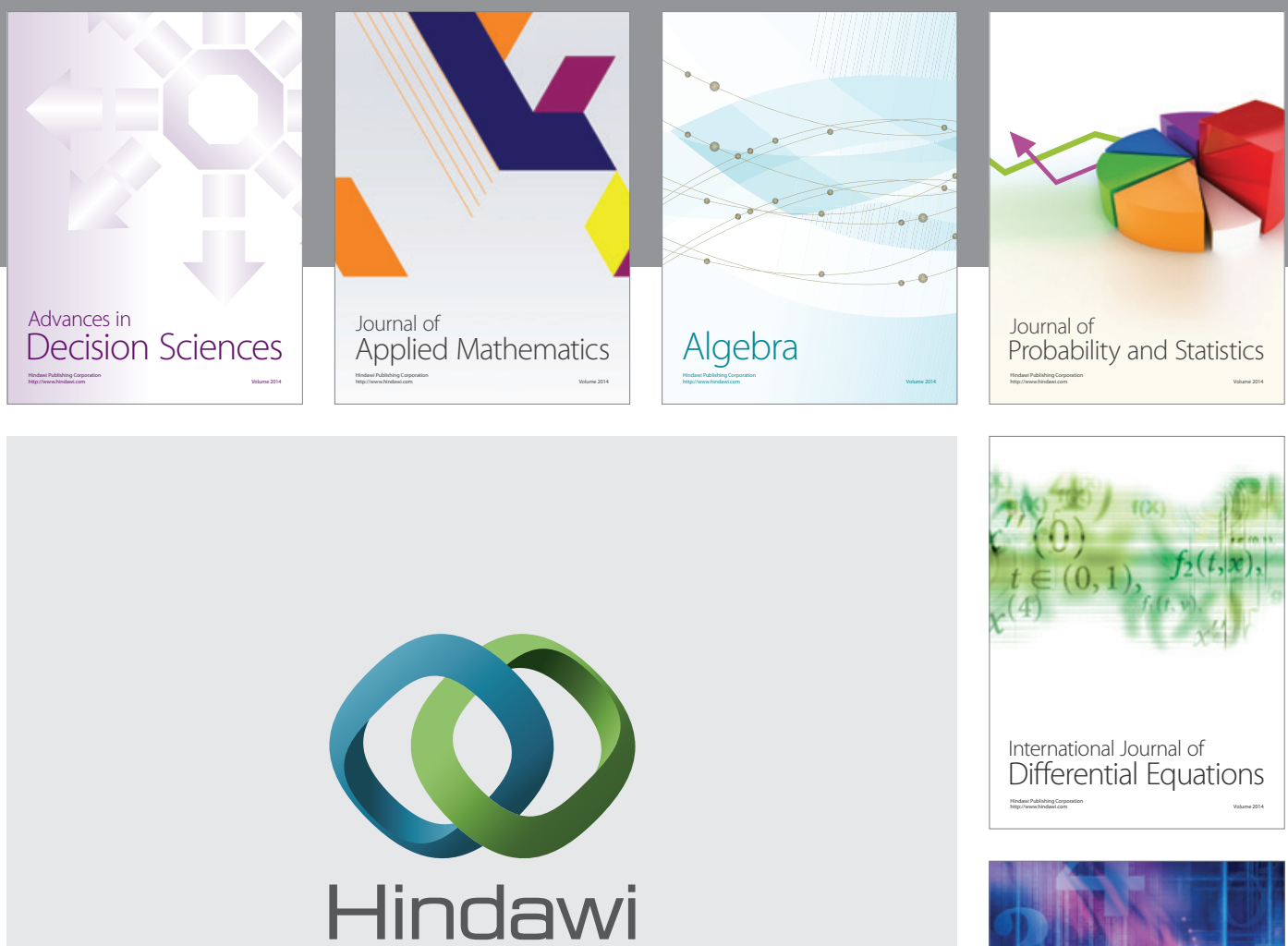

Submit your manuscripts at http://www.hindawi.com
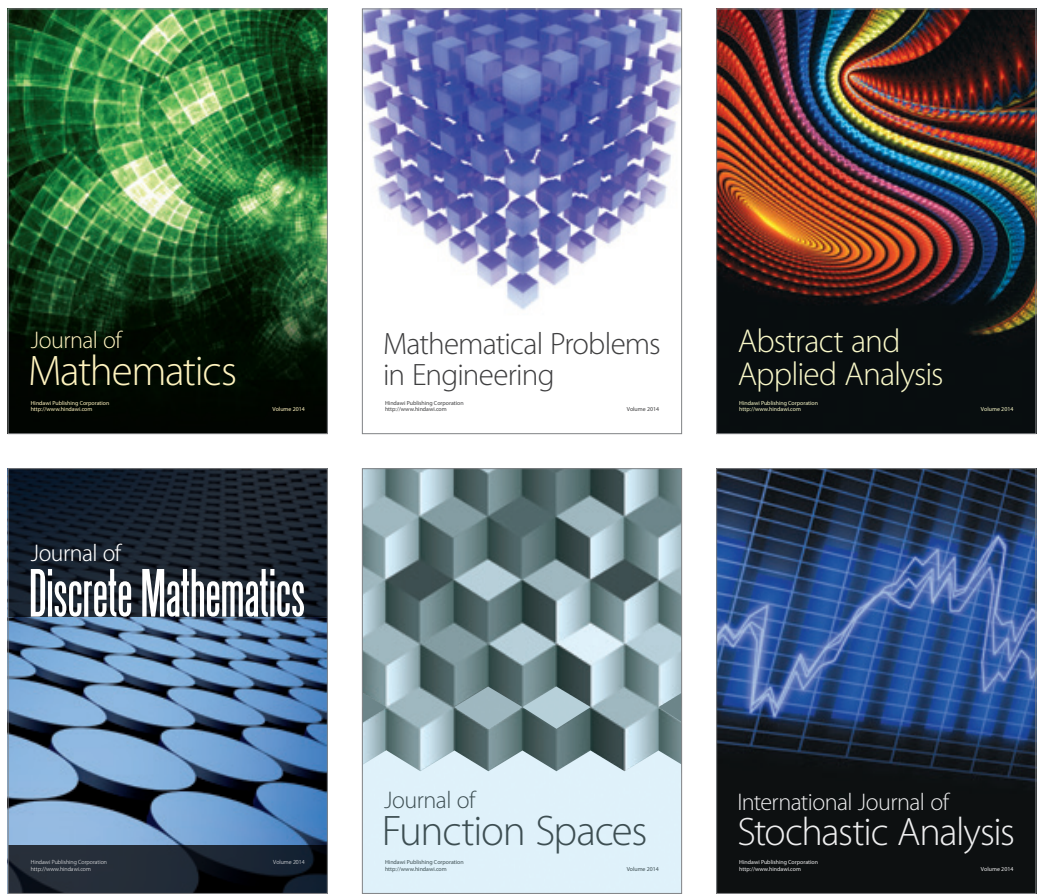

Journal of

Function Spaces

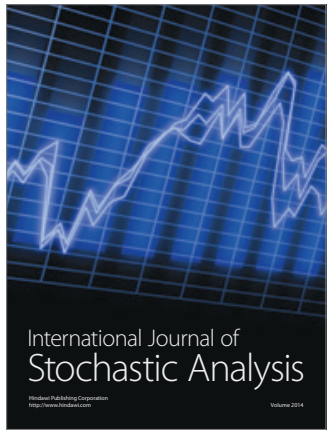

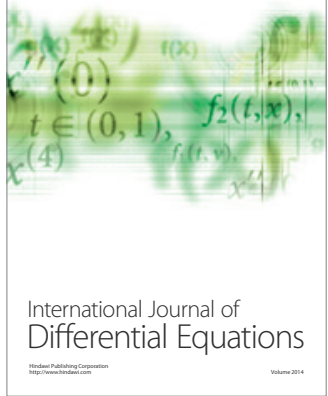
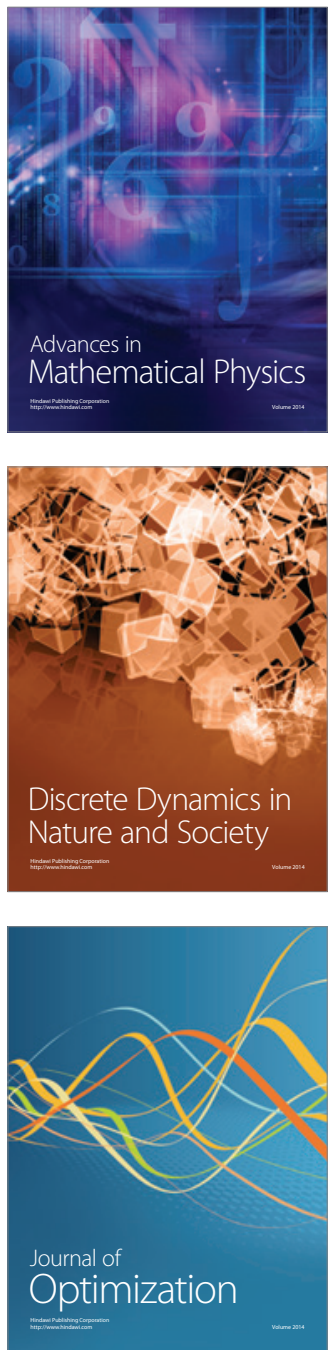